\title{
El método de proyectos como estrategia didáctica
}

\section{The project method as a didactic strategy}

\author{
Edgar Olguín Guzmán ${ }^{a}$, Jorge Martín Hernández Mendoza ${ }^{b}$
}

\begin{abstract}
:
The project method emerges from a vision of education in which students take greater responsibility for their own learning and where they apply, in real projects, the skills and knowledge acquired in the classroom. When the project method is used as a strategy, students stimulate their strongest skills and develop new ones. They are motivated by a love of learning, a feeling of responsibility and effort, and an understanding of the important role they play in their communities. The project method can be defined as a strategy that recognizes that meaningful learning leads students to an inherent learning process, an ability to do relevant work, and a need to be taken seriously.
\end{abstract}

Keywords:

Learning, skills, student, education.

\section{Resumen:}

El método de proyectos emerge de una visión de la educación en la cual los estudiantes toman una mayor responsabilidad de su propio aprendizaje y en donde aplican, en proyectos reales, las habilidades y conocimientos adquiridos en el salón de clase. Cuando se utiliza el método de proyectos como estrategia, los estudiantes estimulan sus habilidades más fuertes y desarrollan algunas nuevas. Se motiva en ellos el amor por el aprendizaje, un sentimiento de responsabilidad y esfuerzo y un entendimiento del rol tan importante que tienen en sus comunidades. El método de proyectos puede ser definido como una estrategia que reconoce que el aprendizaje significativo lleva a los estudiantes aun proceso inherente de aprendizaje, a una capacidad de hacer trabajo relevante y a una necesidad de ser tomados seriamente.

\section{Palabras Clave:}

Aprendizaje, habilidades, estudiante, educación.

\section{Introducción}

En los proyectos de aprendizaje, el conocimiento es construido de forma colectiva entre alumnos y profesores. Además, el aprendizaje basado en proyectos implica formar equipos de trabajo multidisciplinares y como preparar a los estudiantes para un contexto laboral y económico, diverso y global [1].

El aprendizaje colaborativo implica que los estudiantes se ayuden mutuamente a aprender, compartan ideas y recursos, y planifiquen cooperativamente qué y cómo estudiar. Los docentes no dan instrucciones específicas: más bien permiten a los estudiantes elegir y variar sobre lo esencial de la clase y las metas a lograr, de este modo hacen a los estudiantes participar de su propio proceso de aprendizaje [2].

Esta estrategia didáctica presenta un gran potencial para formar estudiantes con capacidad para la resolución de problemas, habilidades para el aprendizaje cooperativo y, aptitudes para la planificación y coordinación de equipos de trabajo colaborativo.

$a$ Profesor-Investigador del Instituto del Ciencias Básicas e Ingeniería. Universidad Autónoma del Estado de Hidalgo. Área académica de Computación y Electrónica--Campus ICBI Carretera Pachuca-Tulancingo Km. 4.5 Col. Carboneras C.P. 42184. ORCID: https://orcid.org/ 0000-0002-9003-6511, Email: eolguin@uaeh.edu.mx

b Profesor Tiempo Completo de la Escuela Superior de Tepeji del Río. Universidad Autónoma del Estado de Hidalgo, Av. del Maestro \#41 Col. Noxtongo 2da Sección, C.P. 42854. ORCID: https://orcid.org/ 0000-0002-9262-4712, Email: jomar_hm@hotmail.com Received: 28/072021/, Accepted: 15/09/2021, Published: 05/12/2021 
Pero ¿Qué son las estrategias didácticas dentro de un proyecto?

Son un procedimiento didáctico mediante el cuál se busca formar competencias en los estudiantes a través de la realización de proyectos de manera individual o por grupos [1].

Para dar un seguimiento a la aplicación del método de proyectos en el proceso de enseñanza aprendizaje es fundamental asignar actividades y responsabilidades de los estudiantes y del docente, es por ello por lo que el trabajar con el método de proyectos supone la definición de nuevos roles donde, [3].

En el alumno el método de proyectos está centrado en el alumno y su aprendizaje, originando que:

- Se sienta motivado ya que él es quien resuelve los problemas, planea y dirige su propio proyecto.

- Dirija por sí mismo las actividades de aprendizaje.

- Se convierta en un descubridor, integrador y presentador de ideas.

- Defina sus propias tareas y trabaje en ellas, independientemente del tiempo que requieren.

- $\quad$ Se muestre comunicativo, afectuoso, productivo y responsable.

- Use la tecnología para manejar sus presentaciones o ampliar sus capacidades.

- Trabaje en grupo.

Trabaje colaborativamente con otros.

- Construya, contribuya y sintetice información.

- Encuentre conexiones interdisciplinarias entre ideas.

El método de proyectos puede darles a los estudiantes una experiencia de aprendizaje más enriquecedora y auténtica que otros modos de aprendizaje porque esta experiencia ocurre en un contexto social donde la interdependencia y la cooperación son cruciales para hacer las cosas. Este contexto permite a los estudiantes prevenir y resolver conflictos interpersonales. En un ambiente de apoyo, los estudiantes ganan la confianza necesaria para desarrollar sus habilidades individuales, preparándolos para el mundo más allá de la escuela [3].

En el docente el método de proyectos es un modelo innovador de enseñanza aprendizaje. El rol del docente en este modelo es muy distinto al que ejercía en la enseñanza tradicional, donde:
- $\quad$ El aprendizaje pasa del docente al alumno, de tal manera que éste pueda hacerse cargo de su propio aprendizaje.

- El docente está continuamente monitoreando la aplicación en el salón de clase, observando qué funcionó y qué no.

- El docente deja de pensar que tiene que hacerlo todo y da a sus estudiantes la parte más importante.

- El docente se vuelve estudiante al aprender cómo los alumnos aprenden, lo que le permite determinar cuál es la mejor manera en que puede facilitarles el aprendizaje.

- El docente se convierte en un proveedor de recursos y en un participante de las actividades de aprendizaje

\section{Aprendizajes que fomenta el método de proyectos}

El método de proyectos al ser una estrategia transdisciplinaria tiene relación con una amplia gama de técnicas de enseñanza aprendizaje, como lo son el estudio de casos, el debate, el aprendizaje basado en problemas, etc. [2].

El trabajar una o más de estas técnicas en conjunto con el método de proyectos crea un ambiente altamente propicio para la adquisición y el desarrollo de conocimientos, habilidades y actitudes en todos los participantes.

Además de los conocimientos propios que de cada materia o disciplina aprenden los estudiantes, adquieren y desarrollan un cúmulo de habilidades y actitudes como son:

- Usar herramientas cognitivas y ambientes de aprendizaje que motiven a los participantes a representar sus ideas.

- Formar sus propias representaciones de tópicos y cuestiones complejas.

- Aprender ideas y habilidades complejas en escenarios realistas.

- Aplicar sus habilidades a una variedad de contextos.

- Construir su propio conocimiento, de manera que sea más fácil para los participantes transferir y retener información.

- Habilidades sociales relacionadas con el trabajo en grupo y la negociación.

Dificultades y barreras dentro del método de proyectos 
Una objeción es que los proyectos pueden gastar grandes cantidades de tiempo de instrucción, reduciendo las oportunidades para otros aprendizajes.

Puede ser difícil obtener evidencia de que los estudiantes han alcanzado los objetivos establecidos.

Los proyectos son vulnerables a la crítica de que los estudiantes pasan la mayor parte de su tiempo llevando a cabo actividades que pueden no estar directamente relacionadas con el tema o no representar nuevos aprendizajes.

\section{Referencias}

[1] Ballenilla, F. (2015). Enseñar investigando. ¿Cómo formar profesores desde la práctica? Editorial Díada. España.

[2] Hernández, J (2017). “Bases teóricas del método por proyectos en la Educación”, Revista Atlante: Cuadernos de Educación y Desarrollo. Recuperado de: https://www.eumed.net/rev/atlante/2017/08/proyectos$\underline{\text { educacion.html }}$

[3] Lacueva, A (2018). La enseñanza por proyectos. ¿Mito o Reto? Revista Iberoamericana de educación (16), pág. 165-190. Recuperado de: http://www.rieoei.org/oeivirt/rie16a09.pdf

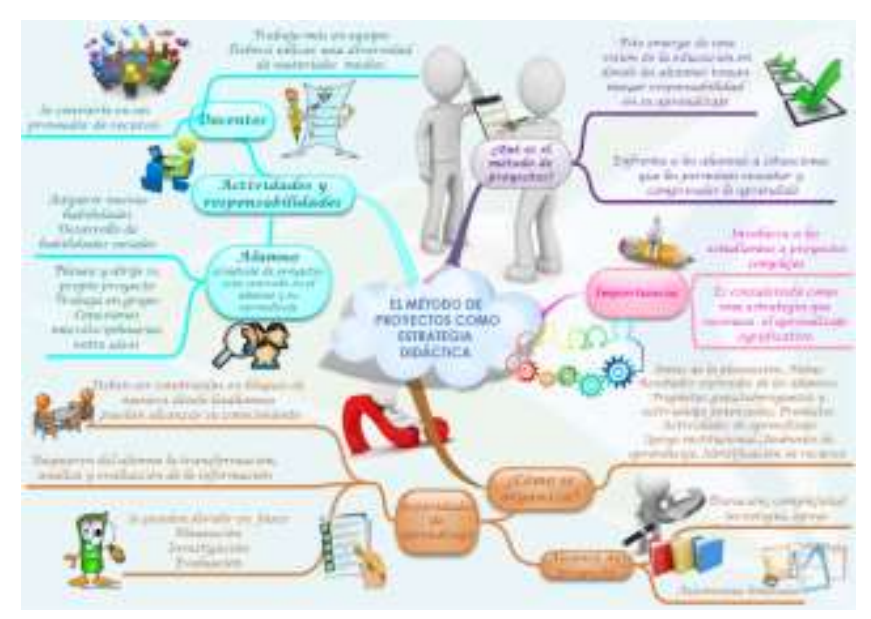

